\title{
Rhabdomyosarcoma of the adult nasopharynx: a case report
}

\author{
Bo-Jiun Chang ${ }^{1}$, Chen-Hsiang Liao ${ }^{1}$, Hsi-Chang Chang ${ }^{1}$, Suat-Yee Lee ${ }^{2}$, Hsin-Lin Chen ${ }^{1}$, \\ Shih-Tsung Chang ${ }^{1}$, Yueh-Chun Lee ${ }^{1,3}$, Ying-Hsiang Chou ${ }^{1,4}$, Hsien-Chun Tseng ${ }^{1,3}$ \\ ${ }^{1}$ Department of Radiation Oncology, Chung Shan Medical University Hospital, Taichung, Taiwan; ${ }^{2}$ Department of Pathology, Chung Shan Medical \\ University Hospital, Taichung, Taiwan; ${ }^{3}$ School of Medicine, Chung Shan Medical University, Taichung, Taiwan; ${ }^{4}$ Department of Medical Imaging \\ and Radiological Sciences, Chung Shan Medical University, Taichung, Taiwan \\ Correspondence to: Hsien-Chun Tseng. Department of Radiation Oncology, Chung Shan Medical University Hospital, No.110, Sec. 1, Jianguo N. Rd., \\ South Dist., Taichung City, 40201, Taiwan. Email: chang.djpa@gmail.com.
}

\begin{abstract}
Rhabdomyosarcomas (RMS) account for 2 to 5 percent of adult soft tissue sarcomas, $40 \%$ of RMS cases arise in adults and 35 to 40 percent of primary sites are in the head and neck. We presented an adult RMS case who underwent chemotherapy and radiotherapy without operation. A 62-year-old woman visited our hospital due to exacerbated left facial and cheek nonpainful mass for 2 weeks. Head and neck MRI revealed a $2.0 \mathrm{~cm} \times 1.3 \mathrm{~cm}$ lesion at the nasopharynx, and multiple enlarged lymph nodes in the neck region. The biopsy of nasopharyngeal tumor revealed RMS. Five cycles of induction chemotherapy were delivered with doxorubicin $\left(30 \mathrm{mg} / \mathrm{m}^{2}\right)$ and Ifosfamide $(50-60 \mathrm{mg} / \mathrm{kg} /$ day), then followed by CCRT 59.4 Gy in 30 fractions to the nasopharynx and regional lymphadenopathies by IMRT with one course of doxorubicin $\left(30 \mathrm{mg} / \mathrm{m}^{2}\right)$ and Ifosfamide $(50-60 \mathrm{mg} / \mathrm{kg} /$ day), and $66 \mathrm{~Gy}$ in 33 fractions (first recurrence at left orbit) and $70 \mathrm{~Gy}$ in 35 fractions (second recurrence at left parotid space) were prescribed with etoposide and cisplatin for twice recurrence of disease. Finally, all of the treatments led to a complete response with limited toxicities. The patient tolerated well during all treatment courses. Our case focused on the good outcome with curative chemoradiation treatment of RMS over nasopharynx, even with twice recurrence over parotid gland and orbital area.
\end{abstract}

Keywords: Radiochemotherapy; rhabdomyosarcomas; nasopharynx

Received: 07 August 2020; Accepted: 29 March 2021; Published: 30 June 2021.

doi: $10.21037 /$ tro-20-52

View this article at: http://dx.doi.org/10.21037/tro-20-52

\section{Introduction}

Rhabdomyosarcomas (RMS) are malignant cells that originate from immature myogenic satellite cells. Accounting for 2 to 5 percent of adult soft tissue sarcomas, $40 \%$ of RMS cases arise in adults and 35 to 40 percent of primary sites are in the head and neck (1). Embryonal and alveolar are two main histologic subtypes. The best policy of the RMS is complete excision with possible pre-or postoperative chemotherapy, and radiotherapy is usually for high risks patients.

However, the current overall treatment outcome of adult head and neck RMS is poor, and the 5-year survival rate is about $8 \%$ (2), and head and neck RMS are rarely amenable to wide local excision because of proximity to vital structures and cosmetic concerns and limit to initial diagnostic biopsy.

We reviewed an adult patient who had RMS of the nasopharynx with unknown histologic subtypes obtained a good outcome after curative treatment of radiotherapy and chemotherapy without surgery.

We presented the following case in accordance with the CARE reporting checklist (available at http://dx.doi. org/10.21037/tro-20-52).

\footnotetext{
^ ORCID: 0000-0001-9961-1802.
} 

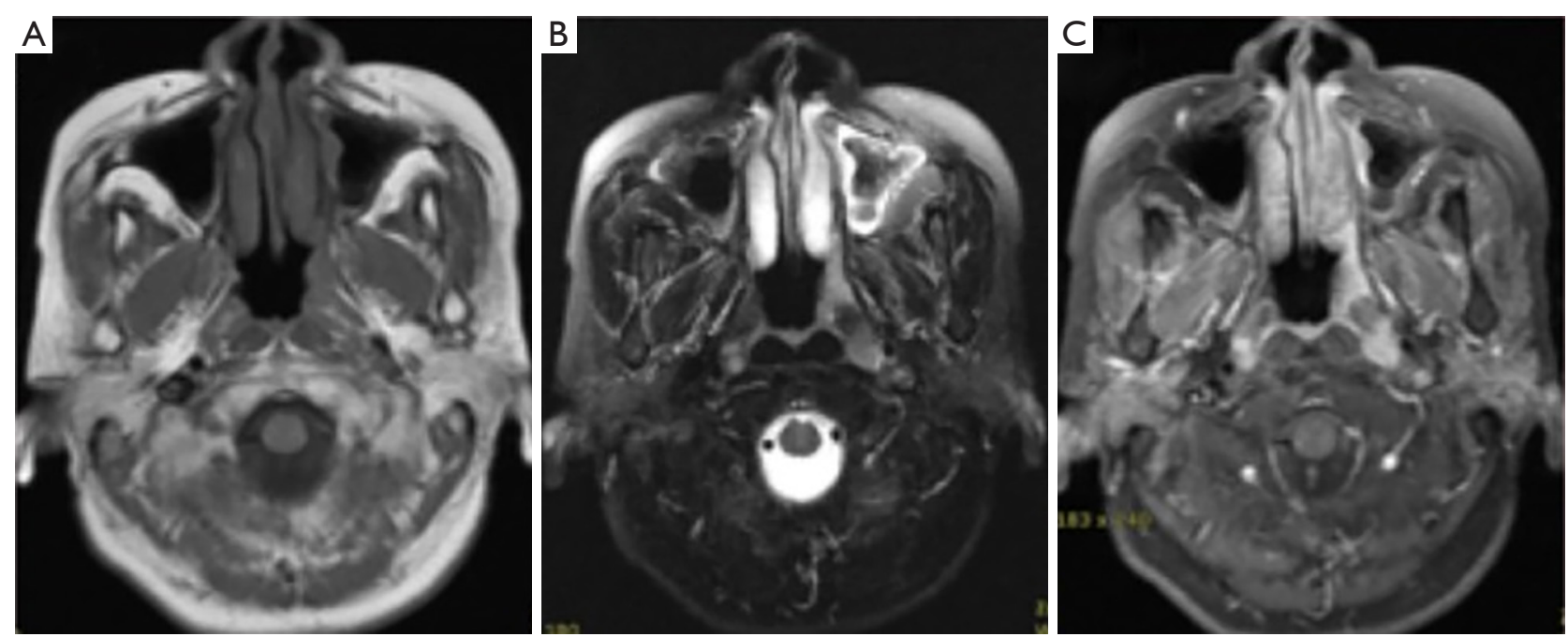

Figure 1 This is a transverse view of patient's head and neck MRI at diagnosis. (A) T1W image (T1 weighted image) (B) the picture showed T2W image (T2 weighted image) (C) the picture showed T1W SPIR image (short tau inversion recovery phase), a $2.0 \mathrm{~cm} \times 1.3 \mathrm{~cm}$ hypointense lesion over the nasopharynx
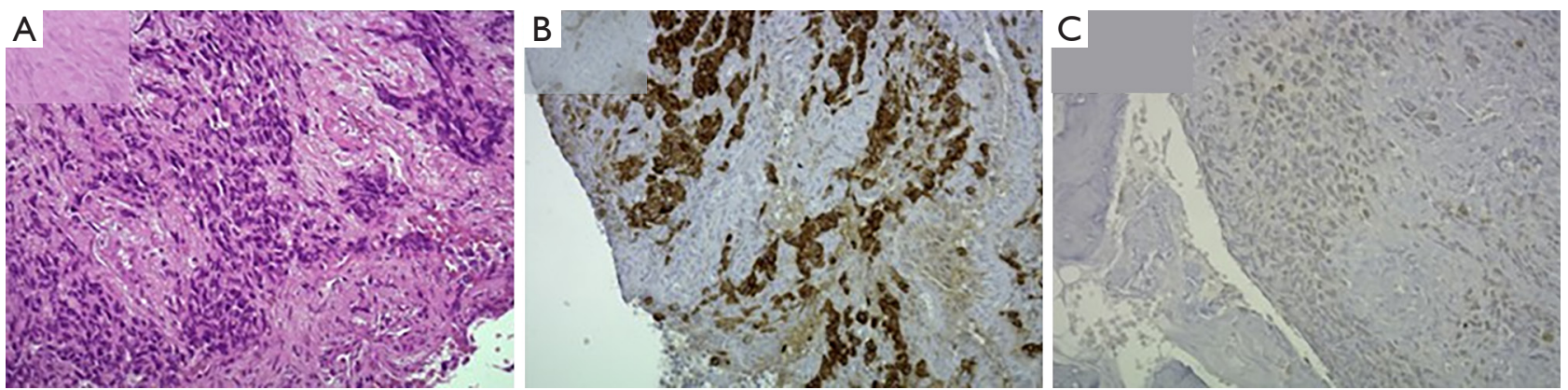

Figure 2 The pathology at diagnosis. (A) The specimen was stained with H\&E, the sections reveal RMS cells in the soft tissue of the nasopharynx (400x). (B) The specimen was stained with desmin(+) (400x). (C) The specimen was stained with myogenin (focal +) (400x).

\section{Case presentation}

A 62-year-old woman visited our hospital due to exacerbated left facial and cheek nonpainful mass for 2 weeks. There was no dyspnea, epistaxis, headache, dizziness, or any other symptoms. The patient does not have any social, family, or medical history. Physical examination showed multiple palpable elastic movable nonpainful masses in the left neck region, 4.5 and $2.3 \mathrm{~cm}$ at level $\mathrm{Ib}-\mathrm{II}, 1.8 \mathrm{~cm}$ at the left supraclavicular area. Head and neck magnetic resonance imaging (MRI) scan revealed a $2.0 \mathrm{~cm} \times 1.3 \mathrm{~cm}$ lesion at nasopharynx, multiple enlarged lymph nodes in the neck region, at left level Ia, left level Ib, left level II, left level III, and left parotid gland (Figure 1).

She received a biopsy of the nasopharynx on Oct. 11,
2012. The pathology report showed rhabdomyosarcoma. RMS is composed of nests of monotonous tumor cells with round nuclei and scant to moderate amount eosinophilic cytoplasm. The crush effect is noted. The immune profiles of tumor cells are CK (-), desmin (+), myoD-1(+), myogenin (+), and S-100(-) (Figure 2).

Under the diagnosis of RMS over left nasopharynx, cT2N1M0, intergroup rhabdomyosarcoma study group (IRSG) III, stage III, high-risk prognosis group. Five cycles of induction chemotherapy were delivered with doxorubicin $\left(30 \mathrm{mg} / \mathrm{m}^{2}\right)$ and Ifosfamide $(50-60 \mathrm{mg} / \mathrm{kg} /$ day) from Oct. 292012 to Jan. 31, 2013. Clinical partial response was obtained. A significant decrease in lesion size of left nasopharynx was noted, with residual $1.3 \mathrm{~cm} \times 0.7 \mathrm{~cm}$ over 


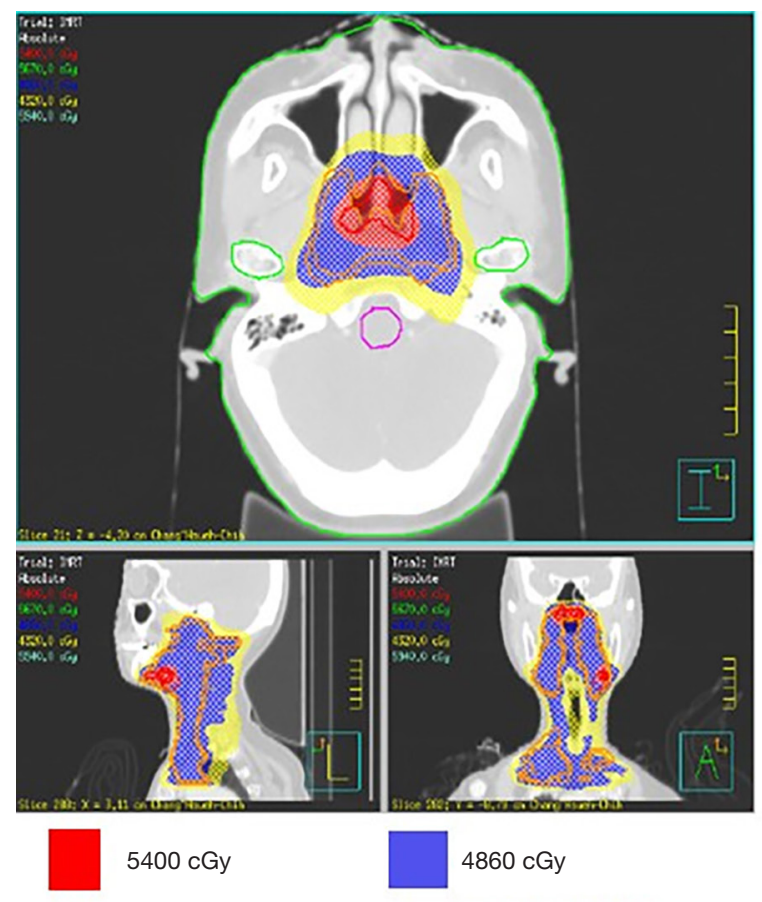

4320 cGy

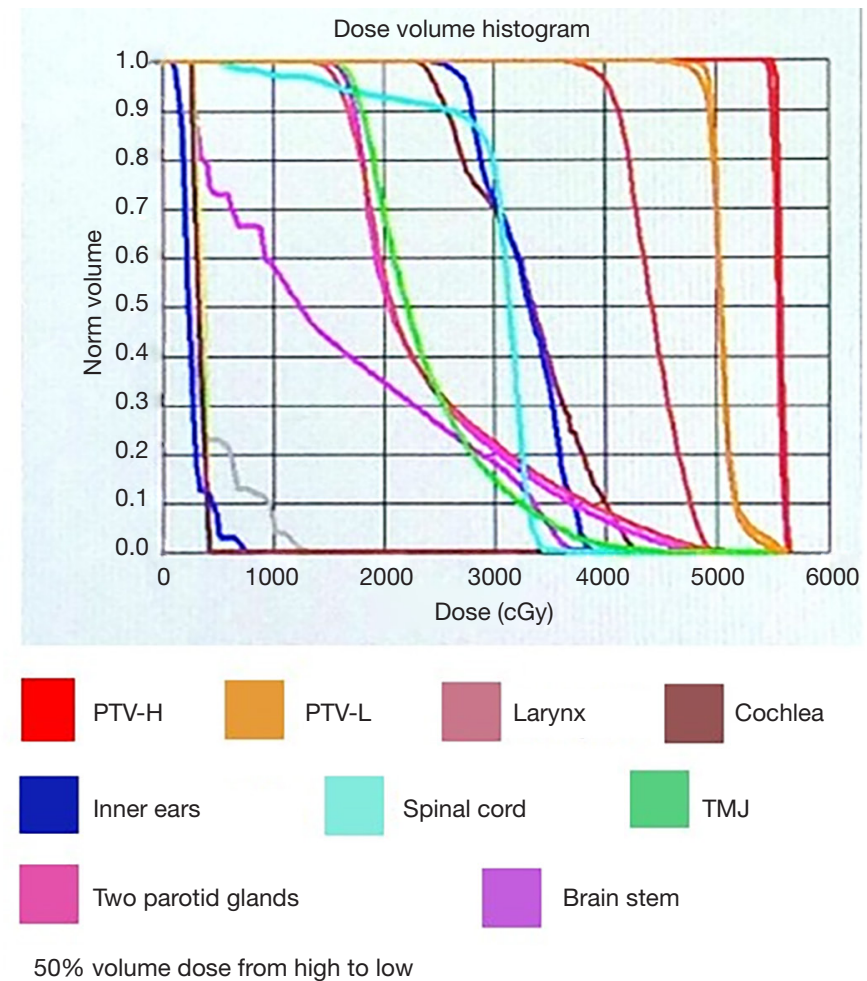

Figure 3 The dose-volume histogram (DVH), region of interest (ROI), and the representative isodose curve of the patient. The radiotherapy plan was delivered using intensity-modulated radiotherapy (IMRT) with a dose of 54 Gy in 27 fractions to nasopharyngeal tumor and regional lymph nodes. The red line of DVH on the right shows 54 Gy to GTV.

left nasopharynx and borderline size $(0.9 \mathrm{~cm})$ lymph nodes over level Ib of left neck. Then concurrent chemoradiation therapy (CCRT) with one cycle of doxorubicin $\left(30 \mathrm{mg} / \mathrm{m}^{2}\right)$ and Ifosfamide (50-60 mg/kg/day) was planned. Computed tomography (CT)-simulation with contrast enhancement in 3-mm-thickness of each slice was performed on Feb. 1st, 2013. The CT images were transferred to the planning system for target delineation. A 3-millimeter margin around the gross target volume (GTV) was included for clinical target volume (CTV). GTV included gross nasopharynx tumor and lymphadenopathies over left level Ib. CTV included nasopharynx area and bilateral level I-V. CTV $+0.3-0.5 \mathrm{~cm}$ was defined as PTV. The dose of 54 Gy in 2 Gy daily fractions to PTV was prescribed by using six megavoltages (MV) photon with intensity-modulated radiotherapy (IMRT) technique (Figure 3). Treatment was administered 5 days per week, for a total of 27 fractions. We followed CWS protocol (3). We prescribed an additional nasopharynx tumor boost 5.4 Gy in 1.8 Gy daily fractions to GTV. Radiotherapy was delivered from Feb 18, 2013, to April 5, 2013.
Concurrent chemotherapy was prescribed once on Mar 5, 2013 (the 12th day of radiotherapy). During treatment, the patient experienced grade 1 oral mucositis. The followup MRI was arranged every 3-6 months after treatment. A complete response was observed by MRI examination in Mar 2013.

However, left orbital tumor recurrence was noted on MRI in October 2013 CCRT was arranged with etoposide $\left(100 \mathrm{mg} / \mathrm{m}^{2}\right)$ and cisplatin $\left(15-20 \mathrm{mg} / \mathrm{m}^{2}\right)$, IMRT was delivered to the gross left orbital tumor (GTV only). PTV was defined as GTV plus $0.3-0.5 \mathrm{~cm}$. The dose of $40 \mathrm{~Gy} / 20$ fractions was prescribed to PTV, followed by left orbital tumor boost $26 \mathrm{~Gy} / 13$ fractions to the residual tumor. Radiotherapy was delivered from Oct 9, 2013, to Nov 25, 2013. Complete response was observed by MRI in 2014 . There was no evidence of tumor recurrence during the regular follow-up until Jun 2014.

Left parotid space tumor recurrence was observed by PET scan. CCRT was arranged with etoposide $\left(100 \mathrm{mg} / \mathrm{m}^{2}\right)$ and cisplatin $\left(15-20 \mathrm{mg} / \mathrm{m}^{2}\right)$, EBRT was delivered with IMRT, GTV included gross left parotid space tumor, CTV 

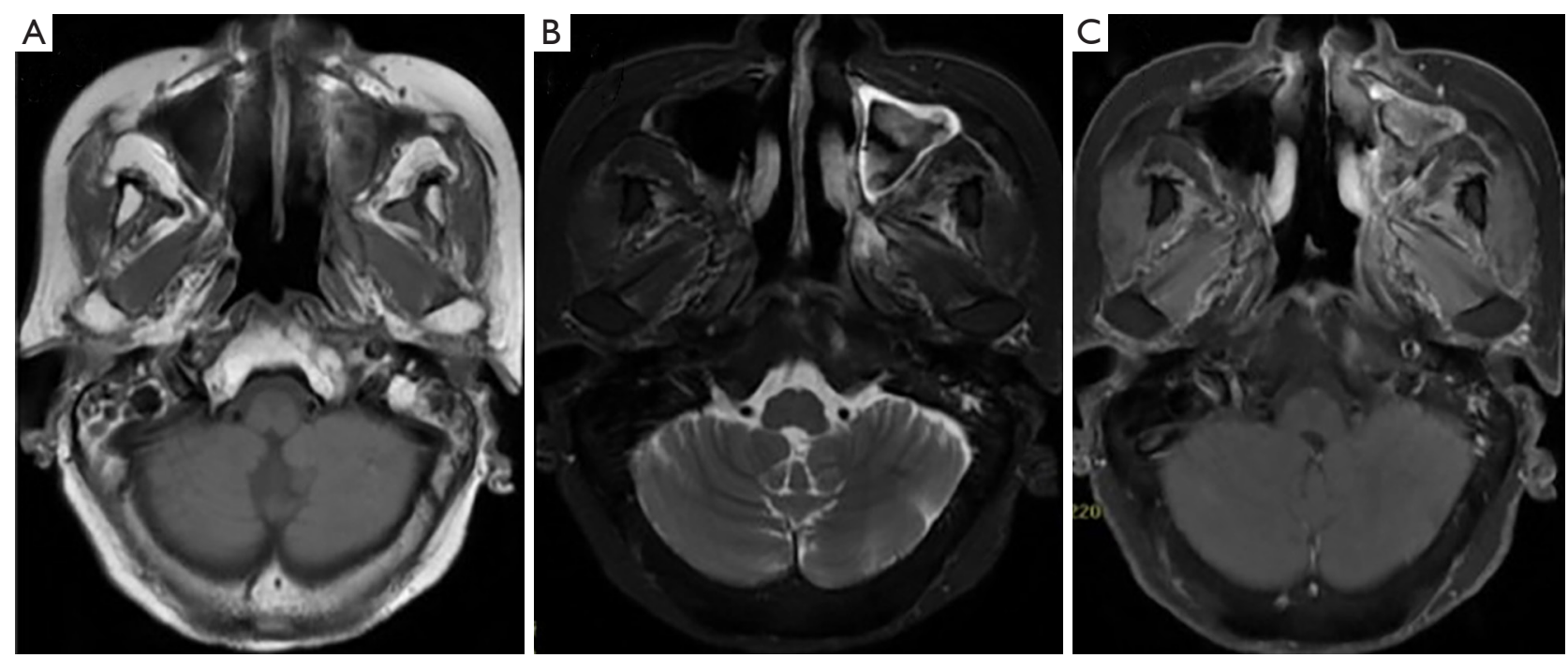

Figure 4 Head and neck MRI on 96th month after treatment. (A) T1W image, (B) T2W image, (C) T1W SPIR image. No significant evidence of abnormal enlargement or abnormal enhancing pattern except left maxillary sinusitis.

equal to GTV. CTV plus $0.3-0.5 \mathrm{~cm}$ was defined as PTV. The dose of $70 \mathrm{~Gy} / 35$ fractions to PTV. was delivered, from July 1, 2014, to Aug 18, 2014.

Complete response was observed once again by MRI in Oct 2014. The patient underwent oral chemotherapy with etoposide 50mg Q7D as maintenance therapy from Aug 2014 to Jun 2018. We had followed up with the patient for 96 months (Figure 4) since the first treatment. She remained free of disease till now.

All procedures performed in studies involving human participants were in accordance with the ethical standards of the institutional and/or national research committee(s) and with the Helsinki Declaration (as revised in 2013). Written informed consent was obtained from the patient

\section{Discussion}

RMS occurred predominantly in children, which arose from myogenic precursor cells. Most cases were less than ten years old. RMS was very uncommon in adults, and highly malignant, accounting for $<1 \%$ of all malignancies.

It differed in adults in terms of presentations, histological distribution, treatment, and outcomes from the pediatric population (4). Adults have a poorer prognosis. The survival varies considerably depending on histological subtypes (5). Head and neck (35\%), pelvic region, and extremities were common sites of RMS occurrence.

Many biomarkers were developed to assist in diagnosing
RMS, including myoglobin, vimentin, troponin, actin, myoglobulin, etc. (6).

Yuhong et al. (7) proved that myogenic regulatory factors (MRFs) including MyoD1, myogenin, Myf5, and MRF4 had positive results in RMS, but negative in other soft tissue tumors. They also suggested that MyoD-1 could be the most efficient biomarker. Based on Immunohistochemistry, our research showed that the expression of desmin, MyoD1 , and myogenin were positive.

Because the crushing effect is noted, cells arranged in sheets and large nests or fibrovascular septae that are lined with densely packed ovoid to round tumor cells and separated by pseudo-alveolar spaces cannot be seen, therefore, lead to an unknown subtype due to the limitation of the biopsy specimen.

Traditionally, the treatment of RMS has been selected according to a risk-based algorithm that combines histologic classification with presurgical stage and postsurgical clinical group.

The best policy of the RMS is complete excision with possible pre-or postoperative chemotherapy. Radiotherapy is usually indicated for high risks patients. However, head and neck RMS are rarely amenable to wide local excision because of proximity to vital structures and cosmetic concerns.

VAC (vincristine, dactinomycin, and cyclophosphamide) are the most commonly used chemotherapeutic agents (8-11). Although in some sequential IRSG and COG 
trials, the addition of many individually active agents (e.g., doxorubicin, cisplatin, etoposide, ifosfamide, topotecan, and melphalan) did not improve outcomes compared with VAC in any subgroup in children (10).

The total dose of radiotherapy is generally 45-60 Gy, and the course of treatment is 5-6 weeks. It was reported that when treating localized parameningeal RMS of children, the dose of $>47.5$ Gy can increase the local control rate of RMS, especially when tumor diameter is $>$ or $=5$ $\mathrm{cm}$ (12). A case report showed a good outcome with $55 \mathrm{~Gy}$ of radiotherapy to lymph nodes, after chemotherapy and human p53 adenovirus injection in an adult patient with nasal embryonic rhabdomyosarcoma stage III (13).

RT should be administered to the primary site, and if there were an intracranial extension, the adjacent meninges should be included. Because of lack of exit dose, proton beam RT can provide superior sparing of normal tissue and a significant reduction in late effects (14).

If there are tumor cells in the cerebrospinal fluid, suggesting diffuse meningeal disease, craniospinal RT is indicated. If there are multiple parenchymal brain metastases and there are no tumor cells in the cerebrospinal fluid, whole-brain RT should be administered.

In our case, an adult RMS over left nasopharynx, cT2N1M0, intergroup rhabdomyosarcoma study group (IRSG) III, stage III, high risk, poor prognosis group, with unknown subtype due to the limitation of the biopsy specimen. 59.4 Gy in 30 fractions was prescribed to nasopharynx with one course of doxorubicin $\left(30 \mathrm{mg} / \mathrm{m}^{2}\right)$ and Ifosfamide (50-60 mg/kg/day) during the first course of treatment. $66 \mathrm{~Gy}$ in 33 fractions was prescribed to left orbit for the first recurrence, and 70 Gy in 35 fractions was prescribed to left parotid space for the second recurrence. with concurrent etoposide and cisplatin for twice recurrence of the disease. All of the treatment finally led to a complete response.

During the treatment courses, grade 1 oral mucositis was noted during the first course of radiotherapy, and grade 1 dermatitis was noted during the third course of radiotherapy. The patient tolerated the treatment well during all treatment courses.

Pathological staging can predict the outcome based on the Intergroup Rhabdomyosarcoma Study Group (IRSG) (15), and the anatomic site was also a significant prognostic indicator (16). The adult RMS five-year overall survival rates are low, ranging from $40 \%$ to $54 \%$ (17). Fiveyear survival rates of adult head and neck RMS is much lower, which is about $8 \%$. The unfavorable primary site, unfavorable histology, and regional and distant spread were more likely to occur in adults and led to a poorer prognosis.

We presented this case focusing on the good outcome with aggressive chemoradiation and maintenance therapy of repeated recurrence of adult nasopharyngeal RMS with limited toxicities.

\section{Acknowledgments}

We thank all the members of the Department of Radiation Oncology of Chung Shan Medical University Hospital for supporting, including Shao-Ti Lee, Wan-Xun Wang, XinYu Lin, Pei-Fang Cai, Hui-Fang Yang, Ya-Fang Ke, XiuMan Zhan, Xin-Yi Zhu, Qi-Wei Chen, Wen-Lan Wang, Yan-Ru Chang, Yan-Mei Huang, Yu-Zhen Xie, Yu-Chan Lu, Shi-Ming Fang, Hsiao-Ju Huang, Ya-Han Chang, YenYu Lo, Qi-Xiang Qu, Yi-Hong Lee, Jiun-hui Wu, Xuan Chang.

Funding: None.

\section{Footnote}

Reporting Checklist: The authors have completed the CARE reporting checklist. Available at http://dx.doi.org/10.21037/ tro-20-52

Conflicts of Interest: All authors have completed the ICMJE uniform disclosure form (available at http://dx.doi. org/10.21037/tro-20-52). The authors have no conflicts of interest to declare.

Ethical Statement: The authors are accountable for all aspects of the work in ensuring that questions related to the accuracy or integrity of any part of the work are appropriately investigated and resolved. All procedures performed in studies involving human participants were in accordance with the ethical standards of the institutional and/or national research committee(s) and with the Helsinki Declaration (as revised in 2013). Written informed consent was obtained from the patient.

Open Access Statement: This is an Open Access article distributed in accordance with the Creative Commons Attribution-NonCommercial-NoDerivs 4.0 International License (CC BY-NC-ND 4.0), which permits the noncommercial replication and distribution of the article with the strict proviso that no changes or edits are made and the original work is properly cited (including links to both the 
formal publication through the relevant DOI and the license). See: https://creativecommons.org/licenses/by-nc-nd/4.0/.

\section{References}

1. Sultan I, Qaddoumi I, Yaser S, et al. Comparing adult and pediatric rhabdomyosarcoma in the surveillance, epidemiology and end results program, 1973 to 2005: an analysis of 2,600 patients. J Clin Oncol. 2009;27:3391-7.

2. Wu TH, Huang JS, Wang HM, et al. Long-term survivors of adult rhabdomyosarcoma of maxillary sinus following multimodal therapy: case reports and literature reviews. Chang Gung Med J 2010;33:466-71.

3. Klingebiel T, Boos J, Beske F, et al. Treatment of children with metastatic soft tissue sarcoma with oral maintenance compared to high dose chemotherapy: report of the HD CWS-96 trial. Pediatr Blood Cancer 2008;50:739.

4. Parham DM, Ellison DA. Rhabdomyosarcomas in adults and children anupdate. Arch Pathol Lab Med 2006;130:1454-65.

5. Parham DM, Barr FG. Classification of rhabdomyosarcoma and its molecular basis. Adv Anat Pathol 2013;20:387-97.

6. Sudhakar S. Alveolar Rhabdomyosarcoma on the Left Maxillary Alveolus: A Unique Presentation. J Clin Diagn Res 2015;9:7-9.

7. Yuhong $M$, Shan $W$, Xiaobing $D$, et al. The expressions and clinical significances of MyoD1 and myogenin in Rhabdomyosarcoma of childhood. J Chongqing Med Univ 2010;12:1870-3.

8. Raney RB, Walterhouse DO, Meza JL, et al. Results of the Intergroup Rhabdomyosarcoma Study Group D9602 protocol, using vincristine and dactinomycin with or without cyclophosphamide and radiation therapy, for newly diagnosed patients with low-risk embryonal rhabdomyosarcoma: a report from the Soft Tissue Sarcoma Committee of the Children's Oncology Group. J Clin Oncol 2011;29:1312.

9. Walterhouse DO, Pappo AS, Meza JL, et al. Reduction of cyclophosphamide dose for patients with subset 2 lowrisk rhabdomyosarcoma is associated with an increased risk of recurrence: A report from the Soft Tissue Sarcoma Committee of the Children's Oncology Group. Cancer 2017;123:2368-75.

10. Arndt CA, Stoner JA, Hawkins DS, et al. Vincristine, actinomycin, and cyclophosphamide compared with vincristine, actinomycin, and cyclophosphamide alternating with vincristine, topotecan, and cyclophosphamide for intermediate-risk rhabdomyosarcoma: children's oncology group study D9803. J Clin Oncol 2009;27:5182.

11. Hawkins DS, Chi YY, Anderson JR, et al. Addition of Vincristine and Irinotecan to Vincristine, Dactinomycin, and Cyclophosphamide Does Not Improve Outcome for Intermediate-Risk Rhabdomyosarcoma: A Report From the Children's Oncology Group. J Clin Oncol 2018;36:2770.

12. Michalski JM, Meza J, Breneman JC, et al. Influence of radiation therapy parameters on outcome in children treated with radiation therapy for localized parameningeal rhabdomyosarcoma in Intergroup Rhabdomyosarcoma Study Group trials II through IV. Int J Radiat Oncol Biol Phys 2004;59:1027-38.

13. He MM, Zhang X, Li P, et al. Embryonic Rhabdomyosarcoma of the Nasopharynx: a Case Report and Literature Review. J Clinc Res Rev 2020;1:3-5.

14. Kozak KR, Adams J, Krejcarek SJ, et al. A dosimetric comparison of proton and intensity-modulated photon radiotherapy for pediatric parameningeal rhabdomyosarcomas. Int J Radiat Oncol Biol Phys 2009;74:179-86.

15. Newton WA Jr, Gehan EA, Webber BL, et al. Classification of rhabdomyosarcomas and related sarcomas. Pathologic aspects and proposal for a new classification-an Intergroup Rhabdomyosarcoma Study. Cancer 1995;76:1073-85.

16. Thompson LDR, Jo VY, Agaimy A, et al. Sinonasal Tract Alveolar Rhabdomyosarcoma in Adults: A Clinicopathologic and Immunophenotypic Study of FiftyTwo Cases with Emphasis on Epithelial Immunoreactivity. Head Neck Pathol 2018;12:181-92.

17. Oberlin O, Rey A, Lyden E, et al. Prognostic factors in metastatic rhabdomyosarcomas: results of a pooled analysis from United States and European cooperative groups. J Clin Oncol 2008;26:2384-9.

doi: $10.21037 /$ tro-20-52

Cite this article as: Chang $\mathrm{BJ}$, Liao $\mathrm{CH}$, Chang $\mathrm{HC}$, Lee SY, Chen HL, Chang ST, Lee YC, Chou YH, Tseng HC. Rhabdomyosarcoma of the adult nasopharynx: a case report. Ther Radiol Oncol 2021;5:10. 